\title{
Correlation between technetium and lithium in a sample of oxygen-rich AGB variables ${ }^{\star}$
}

\author{
S. Uttenthaler ${ }^{1}$ and T. Lebzelter ${ }^{2}$ \\ ${ }^{1}$ Instituut voor Sterrenkunde, K. U. Leuven, Celestijnenlaan 200D, 3000 Leuven, Belgium \\ e-mail: stefan@ster.kuleuven.be \\ 2 Department of Astronomy, University of Vienna, Türkenschanzstraße 17, 1180 Vienna, Austria \\ e-mail: lebzelter@astro.univie.ac.at
}

Received 20 May 2009 / Accepted 27 October 2009

\begin{abstract}
Context. The elements technetium and lithium are two important indicators of internal nucleosynthesis and mixing in late-type stars. Studying their occurrence and abundance can give deep insight into the structure and evolution in the late phases of the stellar life cycle.

Aims. The aims of this paper are: 1) to revisit the Tc content of a sample of oxygen-rich (M-type) asymptotic giant branch (AGB) variables and 2) to increase the number of such stars for which the Li abundance has been measured to provide constraints on the theoretical models of extra-mixing processes.

Methods. To this end, we analysed high-resolution spectra of 18 sample stars for the presence of absorption lines of Tc and Li. The abundance of the latter was determined by comparing the observed spectra to hydrostatic MARCS model spectra. Bolometric magnitudes were established from near-IR photometry and pulsation periods.

Results. We correct the classification with respect to the presence of Tc for a number of stars. We reclassify the star V441 Cyg as Tc-rich, and the unusual Mira star R Hya, as well as W Eri, as Tc-poor. The abundance of Li, or an upper limit to it, was determined for all of the sample stars. In all stars with Tc we also detected Li. Most of them have a Li content slightly below the solar photospheric value, except for V441 Cyg, which is super-Li rich with $\sim 1000$ times the solar abundance. We also found that, similar to Tc, a lower luminosity limit seems to exist for the presence of $\mathrm{Li}$.

Conclusions. We conclude that the higher Li abundance found in the cooler and higher luminosity objects could stem from a $\mathrm{Li}$ production mechanism operating on the thermally pulsing AGB. The stellar mass might have a crucial influence on this (extra mixing) production mechanism. Our findings for R Hya and V441 Cyg have some important consequences for these intriguing objects. It was speculated that the declining pulsation period of R Hya is caused by a recent thermal pulse (TP). While not detecting Tc does not rule out a TP, it indicates that the TPs are not strong enough to drive 3DUP in R Hya. The pieces of evidence concerning the mass of V441 Cyg are not entirely conclusive. It could either be a low-mass, intrinsic S-star that produced its large amount of Li by extra-mixing processes, or an intermediate-mass $\operatorname{star}\left(M \gtrsim 4 M_{\odot}\right)$ undergoing Li production via hot bottom burning.
\end{abstract}

Key words. stars: AGB and post-AGB - stars: abundances - stars: evolution - stars: interiors - stars: individual: R Hya stars: individual: V441 Cyg

\section{Introduction}

The asymptotic giant branch (AGB) phase is the last stage of evolution with nuclear burning for stars of low and intermediate mass $\left(1-8 M_{\odot}\right)$. In the most luminous part of the AGB, the behaviour of an AGB star is characterised by the so-called thermal pulses (TP), thermal instabilities of the He shell accompanied by changes in luminosity, temperature, pulsation period, and internal structure (see e.g. Busso et al. 1999; Herwig 2005, for reviews). Between the repeated events of explosive He-burning, heavy elements can be produced via the "slow neutron capture process" (s-process, see e.g. Wallerstein et al. 1997) in the region between the hydrogen- and the helium-burning shells. The processed material may then be brought to the stellar surface by the convective envelope that temporarily extends to these very deep layers. This mixing event is called the third dredge-up (3DUP)

* Based on observations at the Very Large Telescope of the European Southern Observatory, Cerro Paranal/Chile under Programme 65.L-0317(A), and at the Calar Alto Observatory Centro Astronómico Hispano Alemán, Calar Alto, Spain. and is the cause of the eventual metamorphosis of an oxygenrich M-star into a carbon-rich C-star.

The element technetium (Tc, $Z=43$ ) is a wellestablished diagnostic tool in the study of the thermally pulsing AGB (TP-AGB) phase. Because of the short half life time of the longest lived Tc isotope produced in the s-process $\left({ }^{99} \mathrm{Tc}\right.$, $\tau_{1 / 2}=2.1 \times 10^{5} \mathrm{yr}$ ), any Tc that is detected in the star's atmosphere must have been produced in-situ. This requirement has been used to study the evolution of AGB stars ever since the pioneering discovery of $\mathrm{Tc}$ absorption lines in the spectra of late type giants by Merrill (1952b). Several classes of objects have been investigated for the presence of Tc in the past (e.g. Dominy \& Wallerstein 1986; Little et al. 1987; Van Eck \& Jorissen 1999). A key question in this research is if and when in the evolution on the AGB, in terms of luminosity, the 3DUP sets in. This question was addressed by Lebzelter \& Hron $(1999,2003)$ for a sample of solar-neighbourhood semi-regular pulsating AGB stars and for a larger sample of Galactic disk AGB stars, including several Mira variables. Recently, Uttenthaler et al. (2007a) has investigated a sample of Galactic bulge AGB variables, for which 
the distance and hence luminosity is more precisely known than for their Galactic disk counterparts. The general conclusion of these works is that the lower luminosity limit for 3DUP to set in, as predicted by theoretical evolutionary models of the AGB, is consistent with the lowest luminosity Tc-rich stars. However, being above this limit is not sufficient for a star to have Tc in its atmosphere (Lebzelter \& Hron 2003; Uttenthaler et al. 2007a). In the present paper we revisit the Tc content of a sample of oxygen-rich AGB stars, with important consequences for a few individual stars.

The element lithium ( $\mathrm{Li}, Z=3$ ) is very fragile and temperature-sensitive. Once it is mixed into stellar layers with temperatures exceeding $3 \times 10^{6} \mathrm{~K}$, it is destroyed via proton captures to form helium. Its fragility makes $\mathrm{Li}$ an important diagnostic tool for stellar evolution (Rebolo 1991), and also has a high significance in the determination of cosmological parameters (Spite \& Spite 1982; Korn et al. 2006). The standard model (e.g. Michaud \& Charbonneau 1991) predicts a strong reduction in the Li surface abundance after the first dredge up to values $\log \epsilon(\mathrm{Li})<1.5$, or even less if depletion effects on the main sequence are considered. Most giants for which a Li abundance has been determined (see a summary by Michaud \& Charbonneau 1991) agree with this result.

Despite its fragility, Li can be produced rather than destroyed under certain condition in stars via the so-called CameronFowler or ${ }^{7} \mathrm{Be}$-transport mechanism $\left({ }^{3} \mathrm{He}(\alpha, \gamma)\right)^{7} \mathrm{Be}\left(\mathrm{e}^{-}, v\right){ }^{7} \mathrm{Li}$; Cameron \& Fowler 1971). The production of Li is always connected to mixing mechanisms at or below the bottom of the convective envelope, and requires ${ }^{3} \mathrm{He}$ to be present in the stellar atmosphere. One stage in stellar evolution where Li production occurs is the luminosity bump of the red giant branch (RGB) when the H-burning shell erases the molecular weight discontinuity left behind by the first dredge up. A small fraction of giants in this phase are known to be very Li-rich (de la Reza et al. 1997; Charbonnel \& Balachandran 2000). The mixing process below the convective envelope has been named extra-mixing or cool bottom processing (CBP; Wasserburg et al. 1995). Its effects are also reflected in the carbon isotopic ratio measured from the spectra of giant stars (Gilroy \& Brown 1991), as well as in other isotopic ratios measured in meteoritic pre-solar grains of RGB or AGB origin (Nollett et al. 2003).

The physical cause of the extra-mixing below the convective envelope is hitherto unknown. Eggleton et al. (2006), on the basis of a 3D simulation, find that Rayleigh-Taylor instabilities below the convective envelope can develop from the inversion of the mean molecular weight gradient induced by ${ }^{3} \mathrm{He}$ burning. Alternatively, Charbonnel \& Zahn (2007) suggest that the double-diffusive mechanism called "thermohaline instability" should be at play. In principle, both these phenomena might also occur on the AGB, though detailed models are just emerging (Cantiello \& Langer 2008). Finally, Busso et al. (2007) explore the possibility that circulation of partially processed matter can be accounted for by the magnetic buoyancy induced by a stellar dynamo operating on the RGB and on the AGB. That CBP is probably active on the AGB has been recently demonstrated by Uttenthaler et al. (2007b, 2008).

Among the AGB stars, those with carbon-rich chemistry (C-type, $\mathrm{C} / \mathrm{O}>1$ due to the dredge-up of freshly synthesised ${ }^{12} \mathrm{C}$ ) have been studied more intensively for their Li content than those with oxygen-rich chemistry $(\mathrm{M}$-type, $\mathrm{C} / \mathrm{O}<1)$ in the past. This may partly have a historical reason because of the early detection of carbon stars with a strong Li resonance line (McKellar 1940; Torres-Peimbert et al. 1964), but also an observational reason. Although the molecular line blanketing mainly caused by the $\mathrm{CN}$ and $\mathrm{C}_{2}$ molecules in the spectral vicinity of the $\mathrm{Li}$ line in C-stars is strong, that of the TiO molecule in oxygen-rich stars of the same effective temperature is even more severe. The lower limit of Li abundance that can be measured is thus higher for O-rich stars than for C-rich stars. In both cases, a $\mathrm{Li}$ abundance determination can only be achieved with the help of spectral synthesis techniques including large numbers of molecular lines. Thus, the counterparts to the catalogues of $\mathrm{Li}$ abundance measurements in C-stars (Denn et al. 1991; Boffin et al. 1993) include only relatively small tables of M-type giants (Luck \& Lambert 1982). Even in this last work, a number of the observed stars are supergiants and non-variable giants that are rather in the RGB than in the AGB phase of evolution.

There are, however, M-type AGB stars that are known to be very rich in Li. This type of star has been observed in the Magellanic Clouds (Smith et al. 1995), and very recently also in the Milky Way galaxy (García-Hernández et al. 2007). These objects are intermediate-mass $\left(M \gtrsim 4-8 M_{\odot}\right)$ TP-AGB stars with a convective envelope penetrating the H-burning shell, thus nuclear-burning occurs partly under convective conditions. This process is generally known as hot bottom burning (HBB; Iben 1973). Under these conditions, carbon, on the one hand, is converted into nitrogen, keeping the $\mathrm{C} / \mathrm{O}$ ratio below 1 , and on the other hand ${ }^{7} \mathrm{Li}$ is produced very efficiently by the CameronFowler mechanism. The Li abundance on the surface of HBB stars can be higher than the initial abundance of the interstellar medium from which they formed. We do not intend to deal with this kind of stars in the present paper, but rather with the less massive AGB stars that have not (yet) dredged up enough carbon to have $\mathrm{C} / \mathrm{O}>1$ and are not massive enough to remain O-rich due to the operation of HBB. Nevertheless, by inspecting their bolometric magnitudes, we will check whether our Li-rich objects belong to this group of stars.

The correlation of $\mathrm{Tc}$ and $\mathrm{Li}$ was studied for a sample of Galactic S-type $(C / O \simeq 1)$ stars by Vanture et al. (2007). Uttenthaler et al. (2007b) present Li abundances and their correlation with Tc in a homogeneous sample of O-rich AGB stars located in the Galactic bulge. Here, we want to increase the sample of O-rich AGB stars whose Li abundance has been measured, and study its correlation with the presence of Tc in this type of stars. These measurements will be useful for understanding the interaction between CBP mechanisms and 3DUP in AGB stars.

The paper is structured in the following way. In Sect. 2, the sample and the observations are described and bolometric magnitudes of the sample stars derived; in Sect. 3 we present the analysis of the spectra, results concerning the presence of Tc and the abundance of $\mathrm{Li}$; in Sect. 4 the results are discussed in view of a possible enrichment mechanism of $\mathrm{Li}$, and we take a closer look at two of the sample stars; Sect. 5 summarises the work.

\section{Sample and observations}

The sample used in this study originally goes back to the search for Tc in Galactic-disk AGB variables in the solar neighbourhood, based on parallaxes measured with the Hipparcos satellite (Lebzelter \& Hron 2003). Primary targets for that study were those stars that had not been searched for Tc in the past and objects without a definite decision on their Tc content. The original spectra of that study are reanalysed in this work. An exception here is the star V441 Cyg, whose observation and analysis was initiated by the work of Vanture et al. (2007). A refined method for detecting Tc described in Sect. 3.1 has led to some re-classifications in the sample, which we describe in the present study. 
Table 1. Important characteristics of the sample stars.

\begin{tabular}{|c|c|c|c|c|c|c|c|c|}
\hline Name & Sp. type & Var. type & $P$ P[days] & $\bar{~} K$ [mag] & $\overline{J J-K[\mathrm{mag}]}$ & Ref. & $\overline{M_{\text {bol }}}$ & $\overline{~[12]-[25]}$ \\
\hline U Cet & M4 & $\mathrm{M}$ & 234.76 & $2^{\mathrm{m} .73}$ & $1{ }^{\mathrm{m}} 11$ & 1 & $-4^{m} \cdot 60$ & -1.14 \\
\hline W Cet & S7 & M & 351.31 & $2^{\mathrm{m}} \cdot 14$ & $1 \mathrm{~m} \cdot 23$ & 1 & $-5^{\mathrm{m}} \cdot 07$ & -1.32 \\
\hline V441 Cyg & M4S & SRA & 288.00 & $1 . \mathrm{m} \cdot 69$ & 1. 39 & 3 & $-5^{\mathrm{m}} \cdot 66$ & -1.28 \\
\hline W Eri & M7 & M & 376.63 & $1 \mathrm{~m} .78$ & 1.42 & 1 & $-5^{\mathrm{m}} \cdot 08$ & -0.90 \\
\hline S Gru & M7 & M & 401.51 & 0.54 & 1. 22 & 1 & $-5 \mathrm{~m} \cdot 35$ & -0.87 \\
\hline R Hor & M7 & M & 407.60 & -0.88 & $1 . \mathrm{m} 24$ & 1 & $-5^{m} \cdot 35$ & -0.92 \\
\hline T Hor & M5 & M & 217.60 & $3 \cdot 34$ & 1.13 & 1 & $-4 \cdot 44$ & -1.15 \\
\hline TW Hor & $\mathrm{C} 5(\mathrm{~N})$ & SRB & 158.00 & $0^{\mathrm{m}} \cdot 19$ & 1.46 & 3 & -4.89 & -1.03 \\
\hline R Hya & M7 & M & 388.87 & $-2 \cdot 52$ & $1 . \mathrm{m} 24$ & 1 & -5.28 & -1.08 \\
\hline RU Hya & M6 & M & 331.50 & 1.60 & 1. 18 & 1 & $-5^{\mathrm{m}} \cdot 07$ & -0.74 \\
\hline Y Lib & M5 & M & 275.70 & $3^{\mathrm{m}} \cdot 16$ & 1.21 & 1 & $-4 \cdot 72$ & -0.73 \\
\hline U Mic & M6 & M & 334.29 & 1.80 & 1.22 & 1 & $-5^{\mathrm{m}} \cdot 00$ & -0.69 \\
\hline Y Scl & M5 & SRB & 79.36 & 0.35 & 1.27 & 2 & -4.24 & -0.94 \\
\hline T Tuc & M4 & M & 250.30 & $2 \cdot 96$ & 1. 18 & 2 & -4.58 & -1.08 \\
\hline ER Vir & M4 & SRB & 55.00 & 1.44 & 1. 10 & 4 & $-4^{\mathrm{m}} \cdot 03$ & -1.41 \\
\hline EV Vir & M4 & SRB & 120.00 & $1 . \mathrm{m} 55$ & $1^{\mathrm{m}} \cdot 15$ & 4 & $-5 \mathrm{~m} \cdot 08$ & -1.34 \\
\hline RS Vir & M7 & M & 353.35 & 1.15 & 1.31 & 1 & $-5^{\mathrm{m}} \cdot 09$ & -0.56 \\
\hline S Vir & M7 & M & 378.01 & 0.30 & 1.27 & 1 & $-5^{\mathrm{m}} \cdot 21$ & -0.98 \\
\hline
\end{tabular}

References for near infrared data: 1: Catchpole et al. (1979); 2: Fouquet et al. (1992); 3: Kerschbaum \& Hron (1994); 4: Kerschbaum (1995).

Table 1 lists the 18 objects studied in this project with a number of important characteristics. The spectral type is a kind of "mean" value for what is found in the literature (using Vizier; Ochsenbein et al. 2000) ${ }^{1}$. The carbon star TW Hor is an exception in this sample. We include it because it was observed in the same UVES run as most of the other sample stars. According to the spectral type designations, the stars W Cet and V441 Cyg have a notable enhancement of s-elements in their atmospheres. V441 Cyg is described in the investigation of Galactic S-stars by Vanture et al. (2007), who identify this star as the only one in their sample showing $\mathrm{Li}$ in its spectrum, but not Tc. We come back to this star in Sect. 4.1 where we challenge this classification.

The variability type in Col. 3 of Table 1 is taken from the Combined General Catalogue of Variable Stars (CGCVS), version no. 4.2 (Samus et al. 2004). Also the pulsation period in Col. 4 is taken from the CGCVS, except for Y Scl, for which the period is taken from the All Sky Automated Survey catalogue (Pojmanski et al. 2005), as well as RS Vir and S Vir, for which the period is taken from Templeton et al. (2005). A further exception is V441 Cyg. Because of the importance of this star for the further discussion of the results (see below), we made a check of the period listed in the General Catalogue of Variable Stars $(375$ d). We did a Fourier analysis of the last 3000 days of the AAVSO archive data of this star (Henden 2009) using the program Period04 (Lenz \& Breger 2005). We found a quite wellexpressed peak at $288 \mathrm{~d}$ and a possible second period at $153 \mathrm{~d}$. A typical visual light amplitude of $2 \mathrm{~m} 0$ is found.

The near-IR photometry listed in Cols. 5 and 6 of Table 1 is taken from the references listed in Col. 7. The derivation of the bolometric magnitude in Col. 8 is described in more detail in Sect. 2.1. The IRAS colour listed in Col. 9 is defined as [12] $-[25]=-2.5 \log \left(F_{12} / F_{25}\right)$.

Most of the targets listed in Table 1 were observed in July 2000 with the UVES spectrograph at ESO's Very Large Telescope under Programme 65.L-0317(A). The resolution of these spectra is $R=\lambda / \Delta \lambda \simeq 50000$, and the wavelength coverage is approximately $380-490 \mathrm{~nm}$ in the "blue arm", and $667-1000 \mathrm{~nm}$ in the "red arm" of UVES. These observations are described in Uttenthaler et al. (2007a), and the spectra are

${ }^{1}$ http://webviz.u-strasbg.fr/viz-bin/VizieR analysed for Tc in Lebzelter \& Hron (2003). We thus refer to these papers for more details. The only exceptions to this are the stars W Cet, which is not included in Lebzelter \& Hron (2003), and V441 Cyg, which was observed for the present study in service mode with the FOCES spectrograph (Pfeiffer et al. 1998) mounted on the $2.2 \mathrm{~m}$ telescope of the Calar Alto Observatory in southern Spain on 23 June 2008. The observations were carried out using the CCD chip with $15 \mu \mathrm{m}$ pixel size and a slit width of $100 \mu \mathrm{m}$, which subtends 1 .' 15 on the sky. The chosen setup yields a spectral resolving power of $R \simeq 65000$. The exposure time was two times $1800 \mathrm{~s}$. Because of the high dynamic range of FOCES and the resulting reduction problems with the "red", "green", and "blue" flats, the spectra were reduced with a custom-made IDL routine. The signal-to-noise ratio (SNR) of the spectra is estimated to be in the range 6-10 at the wavelength of the Tc lines, and $\gtrsim 100$ at the wavelength of the $670.8 \mathrm{~nm}$ Li line ${ }^{2}$. As shown by Uttenthaler et al. (2007a), an SNR of 6-10 is sufficient to decide on the presence of Tc based on a flux-ratio method (see next section), as long as the spectral resolution is high enough.

The general appearance of the spectra is dominated by strong bands of the TiO molecule (except of the carbon star TW Hor, of course), clearly verifying their oxygen-rich nature. Some of the stars also show significant bands of the $\mathrm{ZrO}$ molecule, indicating that some s-process enrichment has taken place. Only stars that are identified as Tc-rich (see below) also show bands of $\mathrm{ZrO}$, and conversely no Tc-poor star shows any clear sign of $\mathrm{ZrO}$ absorption bands. Thus, none of our sample stars is extrinsically enriched in s-process elements by, e.g., mass transfer from a binary companion. However, the absorption bands of the $\mathrm{LaO}$ molecule cannot be clearly identified in any of our sample stars, as is sometimes the case in S-type stars.

\subsection{Bolometric magnitude determination}

To derive absolute $K$-magnitudes, we used the $\log P-K$ relations from Ita et al. (1994). For stars classified as Miras we used Ita's relation $\mathrm{C}$ including their separation for targets with $J-K<1.4$ and $J-K>1.4$. For semi-regular variables we used sequence B or $C^{\prime}$. Naturally, this approach includes some uncertainties. In

\footnotetext{
${ }^{2}$ All wavelengths in this paper are expressed in $\mathrm{nm}$ in air.
} 
their Table 3, Ita et al. (1994) list a typical width of about 0.2 of these sequences. This, however, is much less than the uncertainties of absolute $K$-magnitudes derived from parallax measurements, which are only available for a small number of stars in our sample. A larger error comes from the uncertainty of attributing a star to a given pulsation sequence. Several authors agree (Wood et al. 1999; Kiss \& Bedding 2003; Lebzelter et al. 2005) that large amplitude variables, classically dubed Miras, are found on sequence $\mathrm{C}$, which corresponds to fundamental mode pulsation. The risk of a misclassification will thus be low for the Miras. It is much more difficult to attribute the inhomogeneous group of the five semi-regular sample stars correctly. They can be located on any sequence from $\mathrm{A}$ to $\mathrm{C}$, and the definition of sequences $\mathrm{A}, \mathrm{B}$, and $\mathrm{C}^{\prime}$ is less clear. As selection criterion, we chose the light amplitude following the extensive analysis of Kiss \& Bedding (2003) for the LMC. As on average the amplitude becomes smaller from sequence $\mathrm{C}^{\prime}$ to $\mathrm{A}$, we attributed the non-Miras V441 Cyg, Y Scl, and EV Vir to sequence $\mathrm{C}^{\prime}$, TW Hor and ER Vir to sequence B. It is clear that this classification and the derived luminosities have to be taken with some caution. However, because of the small separation of sequences B and $\mathrm{C}^{\prime}$ compared to the difference to sequence $\mathrm{C}$, any misclassification will not change the overall result substantially.

To derive absolute bolometric magnitudes from the absolute $K$-magnitudes, we need to apply a bolometric correction. We adopt the approach outlined in Mekul (2005) using two different relations between blackbody fits and $J-K$ depending on the $K-L$ colour. The inclusion of a second near infrared colour allows for much better treatment of stars at the cool end of the AGB than other formulas found in the literature. Details will be given elsewhere (Kerschbaum et al., in preparation).

We used near-infrared colours from the literature (see Table 1). The $J$ and $K$ band magnitudes were taken from Catchpole et al. (1979), Fouquet et al. (1992), Kerschbaum \& Hron (1994) and Kerschbaum (1995), respectively. All infrared measurements were converted to the 2MASS system using the relations given in Carpenter (2001). Where more than one measurement was found we used an average value. While the $K$-magnitudes used to derive the absolute bolometric magnitudes were calculated from a star's period, the bolometric correction is based on individual near infrared measurements and thus affected by stellar variability. The extensive material presented by Catchpole et al. (1979) allow estimation of the typical uncertainty of the bolometric correction from the variations in the $J-K$ colour. For Miras, this uncertainty can be up to 0.5 . In a few cases no $K-L$ colour was available. We then used an average of the two relations. In all cases the error introduced by this is less than 0.1 . Interstellar extinction has not been taken into account for these rather nearby objects.

Recently, Guandalini \& Busso (2008) have published a new set of bolometric magnitudes for mass-losing AGB stars. As a result they give a $\log P-M_{\text {bol }}$ relation for Miras (see their Fig. A.1). Using this relation we calculated $M_{\text {bol }}$ for the Miras in our sample. In Fig. 1 we compare the results derived from Guandalini \& Busso (2008) with our values of the absolute bolometric magnitude for the stars in common, showing good agreement between the two approaches.

\section{Analysis and results}

\subsection{Analysis with respect to Tc}

The radial velocity $(r v)$ is the first quantity that we determined from our spectra. It was measured from the $\mathrm{TiO}$ band head

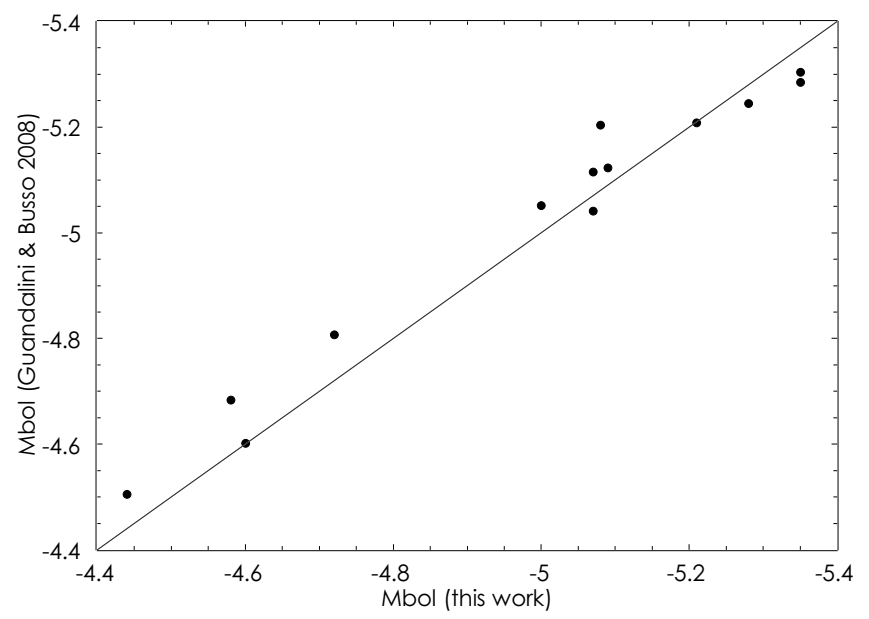

Fig. 1. Comparison of $M_{\text {bol }}$ values derived by out method compared with values using the formula given in Guandalini \& Busso (2008). The full line indicates the 1:1 relation. Good agreement can be seen.

at $705.4 \mathrm{~nm}$ using a cross-correlation technique, with a synthetic MARCS spectrum as template. Sect. 3.2.1 gives more details on the used MARCS models. We chose this TiO feature because the SNR of the observed spectra is much higher at this wavelength than at the wavelength of the Tc lines, and the TiO features are formed in the stellar atmosphere close to the atomic (Tc) lines. For TW Hor, the $r v$ was measured from atomic lines in the vicinity of the Tc lines, due to the absence of TiO bands in carbon stars. The precision of the $r v$ measurement is of the order of $1 \mathrm{~km} \mathrm{~s}^{-1}$ or better. The differences to literature values are usually small (a few $\mathrm{km} \mathrm{s}^{-1}$ ), and can be fully explained by $r v$ variations caused by the stellar pulsation.

We corrected the observed spectra by the measured $r v$ shift to check them for the Tc lines using the flux-ratio method introduced by Uttenthaler et al. (2007a). In this method, the mean flux in a small wavelength interval around a quasi-continuum point close to the Tc line is divided by the mean flux in a small wavelength interval centred on the Tc line itself. This ratio can be calculated for all of the seven identified Tc lines (398.497, 403.163, 404.911 and 409.567, 423.819, 426.227, and 429.706 nm), and plots of the ratios from different lines clearly separate the Tcpoor from the Tc-rich stars. If the Tc lines are absent, the ratio will be close to 1.0 , but significantly more than 1.0 if they are present. Figure 2 shows such a plot for the lines at 423.819 and $426.227 \mathrm{~nm}$ for our sample stars; plots involving other Tc lines are very similar and reveal the same Tc-rich stars. The Tc-rich stars that clearly separate from the Tc-poor ones are identified in the plot with their name. The error bars on the symbols in Fig. 2 were directly derived from the SNR of the observed spectra.

We want to briefly explain the advantages of the flux-ratio method over previous methods of deciding on the presence of Tc. The method previously applied uses the central wavelength of the Tc line blend at $429.706 \mathrm{~nm}$ (e.g. Little et al. 1987; Van Eck \& Jorissen 1999, and references therein). This Tc line is blended with a line of chromium. If Tc is present, the central wavelength is shifted to a somewhat longer wavelength than in the absence of Tc. However, the shift is not large, thus the separation between Tc-poor and Tc-rich stars is not very large either. Every uncertainty of the local wavelength scale has a direct impact on the central wavelength of the blend, thus adding uncertainty to the classification with respect to Tc. Due to the stellar pulsation and the velocity fields in the atmosphere, lines formed at different layers have a slightly different rv shift. This 
S. Uttenthaler and T. Lebzelter: Correlation between Tc and Li in AGB variables

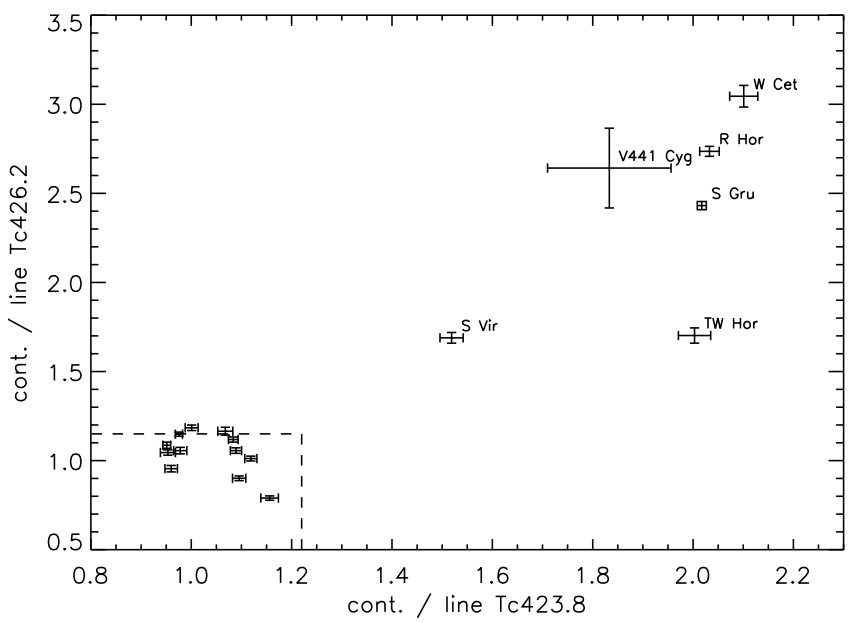

Fig. 2. Ratio of the continuum flux to line core flux for the two Tc lines at 423.819 and $426.227 \mathrm{~nm}$. The quasi-continuum points were taken in the range $423.940-423.970 \mathrm{~nm}$ and $426.130-426.150 \mathrm{~nm}$, respectively, the line core flux was measured in the range $423.805-423.835 \mathrm{~nm}$ and $426.220-426.240 \mathrm{~nm}$, respectively. While the Tc-poor stars gather around ratios 1.0 (lower left corner), the Tc-rich stars are displaced to the upper right by the Tc line absorption. The dashed lines represent the upper limits of the ratios found for the Tc-poor bulge stars analysed in Uttenthaler et al. (2007a).

shift also depends on the pulsation phase at which the observations were done. The result is that intermediate cases are classified as "Tc doubtful", "Tc possible", and "Tc probable". Figure 2 shows that, with our flux-ratio method, the gap between Tc-poor and Tc-rich stars is so large compared to the uncertainty that no room is left for these intermediate cases. The uncertainty of the wavelength scale has only a very small impact on this method. We can thus very clearly decide on "Tc yes" or "Tc no". We conclude that the flux-ratio method delivers more reliable results than the central wavelength method.

Table 2 summarises the radial velocity shifts measured from the spectra and the updated Tc classification. With respect to previous classifications, there are three changes: V441 Cyg is Tc-rich (Vanture et al. 2007, Tc-poor), W Eri is Tc-poor (Lebzelter \& Hron 2003, Tc possible), and R Hya is Tc-poor as well (Lebzelter \& Hron 2003, Tc-rich).

\subsection{Analysis with respect to $\mathrm{Li}$}

The abundance of $\mathrm{Li}$ in stars is usually measured from the strong resonance doublet of neutral $\mathrm{Li}$ at $670.8 \mathrm{~nm}$. The line strength is quite sensitive to the effective temperature of the star, thus a good knowledge of this stellar parameter is required for reliable abundance determination. In the following we describe in some detail how the temperature determination was accomplished for the present sample of stars.

\subsubsection{Effective temperature determination}

For determining the effective temperatures of the sample stars, we applied a $\chi^{2}$ minimisation method to band heads of the TiO molecule using a small grid of synthetic model spectra. In the optical range, $\mathrm{TiO}$ bands are the dominant spectral features of cool oxygen-rich giants, and they are very sensitive to the stellar temperature. We thus followed a procedure similar to García-Hernández et al. (2007), who apply this method to measure Li abundances in massive Galactic AGB (OH/IR) stars.
Table 2. Quantities measured from the spectra: radial velocity, Tc content, effective temperature, and $\mathrm{Li}$ abundance of the sample stars.

\begin{tabular}{lrccr}
\hline \hline Name & $r v\left[\mathrm{~km} \mathrm{~s}^{-1}\right]$ & Tc? & $T_{\text {eff }}$ & $\log \epsilon(\mathrm{Li})$ \\
\hline U Cet & -19.0 & no & 3300 & $\lessgtr 0.0$ \\
W Cet & +5.8 & yes & 3100 & +0.8 \\
V441 Cyg & -61.7 & yes & 3400 & +4.6 \\
W Eri & +17.8 & no & 2950 & +0.4 \\
S Gru & -18.4 & yes & 2900 & +0.8 \\
R Hor & +56.0 & yes & 2900 & +0.6 \\
T Hor & +44.9 & no & 3300 & $\lessgtr 0.0$ \\
TW Hor & +14.5 & yes & 3000 & -1.5 \\
R Hya & -15.7 & no & 3100 & $\lessgtr 0.0$ \\
RU Hya & +0.4 & no & 3300 & $\lessgtr 0.0$ \\
Y Lib & +9.3 & no & 3100 & +0.9 \\
U Mic & -61.0 & no & 2900 & +0.6 \\
Y Scl & +30.0 & no & 3200 & +1.0 \\
T Tuc & -37.9 & no & 3300 & $\lessgtr 0.0$ \\
ER Vir & +8.5 & no & 3500 & $\lessgtr 0.0$ \\
EV Vir & -44.5 & no & 3500 & $\lessgtr 0.0$ \\
RS Vir & -15.9 & no & 2900 & +1.0 \\
S Vir & +7.2 & yes & 2800 & +0.5 \\
\hline
\end{tabular}

We used the same modified $\chi^{2}$ test as these authors to fit model data $Y_{\mathrm{synth}, i}$ to observed data $Y_{\mathrm{obs}, i}$, which is expressed in the form

$\chi^{2}=\sum_{i=1}^{N} \frac{\left[Y_{\mathrm{obs}, i}-Y_{\mathrm{synth}, i}\left(x_{1} \ldots x_{M}\right)\right]^{2}}{Y_{\mathrm{obs}, i}}$,

with $N$ the number of data points and $M$ the number of free parameters. The synthetic spectra are based on COMARCS atmospheric models. COMARCS is a revised version of MARCS (Jørgensen et al. 1992) with spherical radiative transfer routines from Nordlund (1984) and new opacity data from the COMA program (Aringer 2000). The only model parameters that we varied in this procedure were the effective temperature and the metallicity. The other stellar parameters have a very limited impact on the spectral appearance, hence on the results of this analysis (see also García-Hernández et al. 2007, for a more detailed discussion). For the surface gravity $\log g\left[\mathrm{~cm} \mathrm{~s}^{-1}\right]$, microturbulent velocity $\xi$, and the mass $M$, we chose values typical for red giant stars of $0.0,3 \mathrm{~km} \mathrm{~s}^{-1}$, and $1 M_{\odot}$. Since the precise $\mathrm{C} / \mathrm{O}$ ratio cannot be determined from our optical data (near-IR spectra would be required to do so), we fixed it at the solar value of 0.48 , although in principle it might deviate from that value, particularly for the Tc-rich sample stars. Once the $\mathrm{C} / \mathrm{O}$ ratio approaches 1 , the chemistry and thus the dominant features in the spectrum will change significantly. Two stars of our sample are classified as MS or S-type (W Cet and V441Cyg), and only for these objects do we expect that the assumption of a constant $\mathrm{C} / \mathrm{O}$ might deteriorate the results to some degree. We come back to this point in the next section.

We chose three metallicity values for the grid of model atmospheres: $0.3,1.0$, and 1.6 times the solar metallicity. The temperature ranges between 2600 and $3600 \mathrm{~K}$ in steps of $100 \mathrm{~K}$, with intermediate steps at 2950 and $3150 \mathrm{~K}$ for the solar metallicity models. From this grid of model atmospheres, synthetic spectra were calculated in the two spectral pieces $668-674 \mathrm{~nm}$ (covering the $\mathrm{Li}$ line at $670.8 \mathrm{~nm}$ and the $\mathrm{TiO} \gamma(1,0) \mathrm{Rc}$ and $\mathrm{TiO} \gamma(1,0) \mathrm{Rb}$ band-heads) and $700-710 \mathrm{~nm}$ (covering the strong TiO $\gamma(0,0) \mathrm{Ra}$ band-head at $\sim 705 \mathrm{~nm}$ and the TiO $\gamma(0,0) \mathrm{Rb}$ band head). Atomic lines from the VALD database (Kupka et al. 1999) and TiO lines from the list of Schwenke (1998) were taken into account in the spectral synthesis. Other molecular line data of 
less impact on the spectral ranges under consideration were also taken into account; these data are summarised in Table 1 of Cristallo et al. (2007).

Prior to the $\chi^{2}$ minimisation fitting, the model spectra were convolved with a Gaussian to a resolution of $R=\lambda / \Delta \lambda=50000$ (except for V441 Cyg, for which the spectra are convolved to $R=65000$ ). An additional macro-turbulence of $3 \mathrm{~km} \mathrm{~s}^{-1}$ was assumed. This proves an acceptable value for all except two stars, namely V441 Cyg and Y Scl, for which a higher value of $6 \mathrm{~km} \mathrm{~s}^{-1}$ had to be adopted to make a satisfactory fit to the spectra.

Although the metallicities adopted for the atmospheric model grid cover a quite wide range, it turned out that the temperature at which $\chi^{2}$ becomes minimal does not strongly depend on the metallicity. The $\chi^{2}$ minimum for the spectral piece including the Li line is quite flat for many stars, thus the adopted temperature was mostly fixed by the second piece between $700-710 \mathrm{~nm}$. For the spectral synthesis to determine Li abundances (see next section), we therefore adopted the model with solar metallicity, with a temperature that satisfactorily fits both spectral pieces. There is some indication in the literature that solar metallicity is a good assumption for solar neighbourhood AGB stars (e.g. Vanture \& Wallerstein 2002; García-Hernández et al. 2007). After finishing this procedure, we found that the adopted effective temperature correlates well with the $(J-K)$ colour, as can be expected: cooler stars have higher (redder) $(J-K)$ colour than hotter stars. The adopted temperatures are summarised in the fourth column of Table 2. We estimate that the precision of the temperature determination is $\pm 100 \mathrm{~K}$.

\subsubsection{Li abundances}

With this temperature, spectral synthesis calculations of the $\mathrm{Li}$ line doublet were performed to measure the $\mathrm{Li}$ abundance, using the MARCS models with solar metallicity. The $\log g f$ values from the VALD database were adopted for the $670.8 \mathrm{~nm}$ Li doublet $(\log g f=-0.009$ and -0.309 for the component at the shorter and longer wavelength, respectively). The Li abundance was varied in steps of $0.1 \mathrm{dex}$, and the observed-minuscalculated flux at the wavelength of the Li line was monitored to determine the Li abundance in the atmosphere of each sample star. The abundance that left practically a zero flux difference was finally adopted. The last column of Table 2 gives the measured $\mathrm{Li}$ abundances on the $\log \epsilon$ scale $(\log \epsilon(\mathrm{Li})=$ $\log N(\mathrm{Li}) / N(\mathrm{H})+12)$. The solar photospheric Li abundance on this scale is $\log \epsilon(\mathrm{Li})=+1.1$. We also used our model spectra to verify that the observed absorption around the Li line cannot come from a line of cerium, which is observed to be a "substitute" for $\mathrm{Li}$ in the hotter barium stars and post-AGB stars (Lambert et al. 1993; Reyniers et al. 2002).

From the uncertainty of the temperature, the stellar parameter with the strongest influence on the Li line strength, we estimate an abundance uncertainty of \pm 0.4 dex. Taking the uncertainties of the other stellar parameters into account, which are harder to quantify, the total uncertainty on $\log \epsilon(\mathrm{Li})$ is estimated to be up to $\pm 0.6 \mathrm{dex}$, being larger for the cooler stars. The relative uncertainty within the sample, however, is probably less than this.

A number of stars in the sample has no significant Li line absorption, their spectra are satisfyingly fit with negligible Li abundance in the spectral synthesis. It is difficult to state a precise upper limit to their atmospheric $\mathrm{Li}$ abundance. In the vicinity of the Li line, positive and negative residuals are present in an observed-minus-calculated flux plot, due to imperfect spectral modelling. Besides errors on the adopted stellar parameters, neglecting velocity fields in the atmosphere (due to stellar pulsations) by using hydrostatic models leads to persistent observedminus-calculated flux residuals. Also, the line lists (mostly TiO) are certainly not without flaws at the high resolution of our spectra. The stars with no detectable Li line absorption are listed with $\log \epsilon(\mathrm{Li}) \lesssim 0.0$ in Table 2 , an abundance that would certainly not be detectable with our methods anymore. The carbonstar TW Hor was analysed by Kipper (2004), who determine a $\log \epsilon(\mathrm{Li})=-1.5$ from another UVES spectrum of this object. We adopt this value of the Li abundance for TW Hor in Table 2. We also compared our spectrum of TW Hor with the one used by Kipper (2004) and found negligible variation between them. The low Li abundance of the carbon-star TW Hor would not be detectable in oxygen-rich stars of the same effective temperature, because of the increased molecular absorption in M-type stars in this spectral region. In the following we focus on the oxygen-rich stars. Figure 3 shows sample spectra of two stars (R Hya and RS Vir) with very different Li abundances, along with a best-fitting model spectrum. The Li content of R Hya is below the detection threshold, whereas RS Vir is found to have Li at the solar photospheric level.

The very high Li abundance of V441 Cyg is remarkable. As noted above, this star deviates from the trend towards higher (redder) $J-K$ colours at lower temperatures: despite its rather high temperature estimate of $3400 \mathrm{~K}$, a $J-K=1.39$ is found. Vanture \& Wallerstein (2002) estimate $T_{\mathrm{eff}}=3100 \mathrm{~K}$ from its spectral type. This low temperature is hardly compatible with our fit to the $700-710 \mathrm{~nm}$ spectral region. While there is some disagreement on the near-IR photometry (Wang \& Chen 2002), the combination of red $J-K$ but weak TiO bands could be caused by a high $\mathrm{C} / \mathrm{O}$ ratio. From our models we found that a synthetic spectrum with $\mathrm{C} / \mathrm{O}=0.9$ and a temperature of $3200 \mathrm{~K}$ also reasonably fits the $700-710 \mathrm{~nm}$ region. We thus repeated the $\mathrm{Li}$ abundance measurement adopting $\mathrm{C} / \mathrm{O}=0.9$ and $T_{\text {eff }}=3200 \mathrm{~K}$ and derived $\log \epsilon(\mathrm{Li})=4.1$, which is still a factor of 1000 higher than the solar photospheric abundance! However, Plez et al. (2003) demonstrate that atomic and some molecular lines (such as from $\mathrm{ZrO}$ and $\mathrm{LaO}$ ) strengthen even more when the $\mathrm{C} / \mathrm{O}$ ratio approaches 1 . To find the $\mathrm{Li}$ abundance that may be regarded as a lower limit, we constructed a MARCS model with a C/O ratio of 0.99 and $T_{\text {eff }}=3400 \mathrm{~K}$, but otherwise unaltered stellar parameters. Using this model, we find $\log \epsilon(\mathrm{Li})=3.1$ for V441 Cyg. The TiO bands are still well fit by the model at this high a $\mathrm{C} / \mathrm{O}$ ratio.

The equivalent width of the Li I $670.8 \mathrm{~nm}$ line in V441 Cyg is approximately $730 \mathrm{~m} \AA$, surpassing that of many of the superLi rich stars identified in the Magellanic Clouds (Smith et al. 1995 ), and the line core flux is $\sim 6 \%$ of the local quasicontinuum level. Also, the Li line at $812.634 \mathrm{~nm}$ was identified in V441 Cyg. A spectrum synthesis of this line yielded a Li abundance lower by 0.8 dex (at solar $\mathrm{C} / \mathrm{O}$ ) than was found from the $670.8 \mathrm{~nm}$ Li line. However, the spectral fit in the vicinity of the $812.6 \mathrm{~nm}$ line was not as good as that around the $670.8 \mathrm{~nm}$ line, which caused problems in defining the quasi-continuum level.

We repeated the $\mathrm{Li}$ abundance measurement with the $\mathrm{C} / \mathrm{O}$ ratio increased to 0.99 for W Cet, the other S star in our sample. This exercise yielded $\log \epsilon(\mathrm{Li})=-2.0$, which may again be regarded as a lower limit for the $\mathrm{Li}$ abundance. The Li detection would still be significant, because the detection threshold will also decrease markedly with $\mathrm{C} / \mathrm{O}$ approaching 1 . The observed spectrum of W Cet is still incompatible with negligible Li content, if $\mathrm{C} / \mathrm{O}=0.99$ is adopted for the model atmosphere. For 


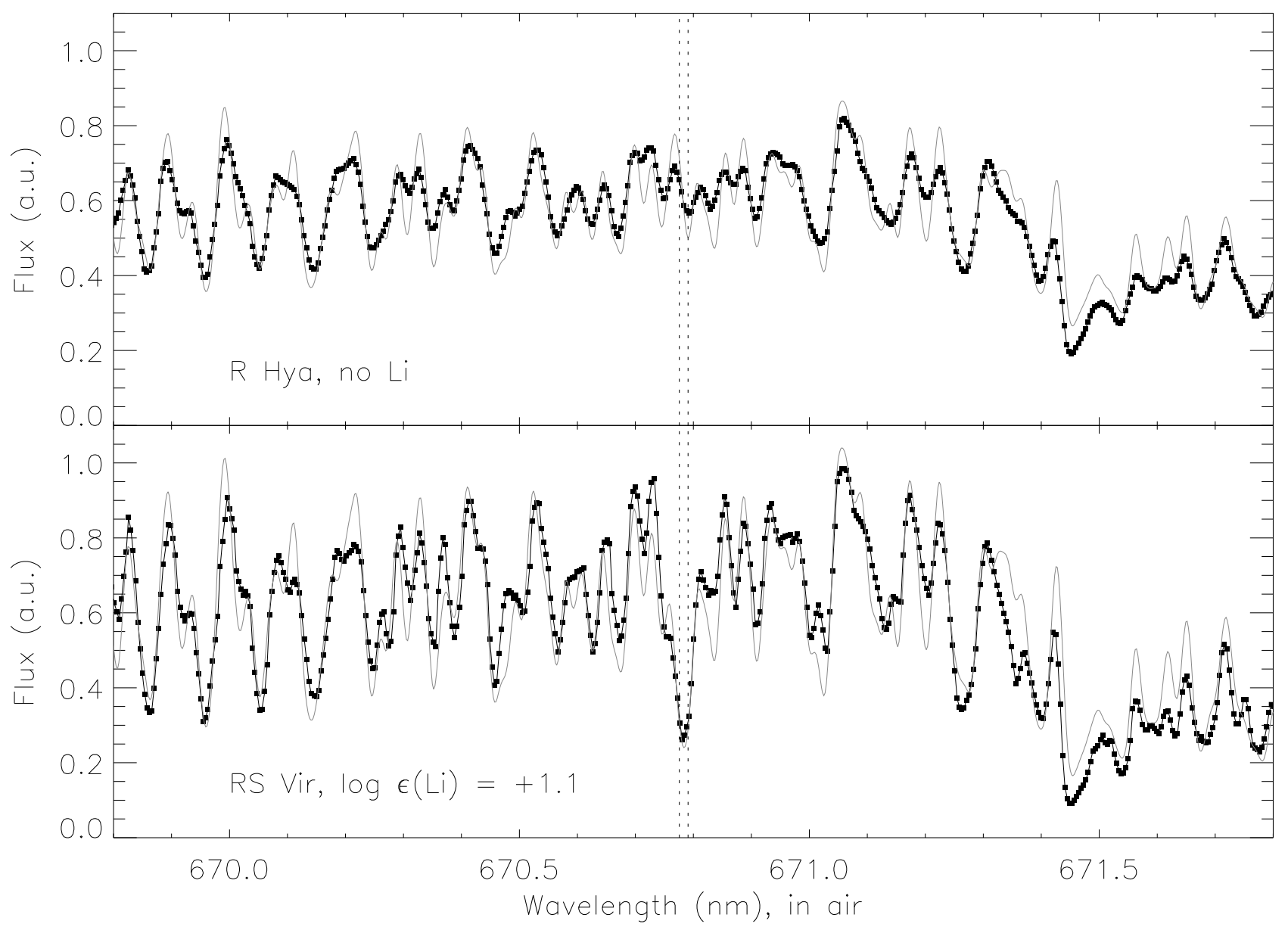

Fig. 3. Examples of observed spectra (black graph with squares) of two stars with very different Li abundances, along with a best-fitting model spectrum (grey graph). The dashed lines indicate the laboratory wavelengths of the Li doublet.

consistency, we keep the Li abundances as determined with the $\mathrm{C} / \mathrm{O}=0.48$ models in Table 2 and in the analysis.

We would also like to note that we did not identify significant $\mathrm{LaO}$ bands in any of our oxygen-rich stars, thus we may assume that indeed none of them has a C/O ratio above 0.99 .

Besides V441 Cyg, only one of our sample stars has previously been investigated for its Li content, namely RS Vir (García-Hernández et al. 2007). In contrast to our measurement (Table 2), García-Hernández et al. (2007) find no significant Li line absorption in that star, and state an upper limit of $\log \epsilon(\mathrm{Li})<0.5$ instead. Also, the temperature determinations of this star differ somewhat, $2900 \mathrm{~K}$ in the present work compared to $2700 \mathrm{~K}$ in García-Hernández et al. (2007). We identify two possible explanations for this discrepancy.

First, two different TiO line lists were applied to derive stellar temperatures. One of them might yield systematically lower temperatures, which would decrease the measured $\mathrm{Li}$ abundance considerably. To investigate this possibility, we calculated model spectra using the TiO line list of Plez (1998), as used by García-Hernández et al. (2007), and repeated the $\chi^{2}$ minimisation procedure (Sect. 3.2.1). Only the sequence of models with solar metallicities was used for this. From this we find that the temperatures found using the two TiO line lists hardly differ from each other, with the Plez list delivering temperatures at most $50 \mathrm{~K}$ higher than the Schwenke list. We conclude that the different line lists used cannot explain the discrepancy found in the temperature and Li content of RS Vir. From this experiment with the two TiO line lists, we also find that the list of
Schwenke (1998) yields slightly lower residuals than that of Plez (1998), particularly in the vicinity of the $670.8 \mathrm{~nm} \mathrm{Li}$ line. This might also explain why our detection limit is somewhat lower than that of García-Hernández et al. (2007).

Second, the two spectra have been taken at different epochs, with different instruments. The temperature variation over the cycle of stellar pulsation could be responsible for the difference of $200 \mathrm{~K}$ found by the two studies. According to the AAVSO visual light curve, our spectrum was taken just 30 days before maximum, when the light curve was still sharply rising. García-Hernández et al. (2007) observed RS Vir during their fourth run between 21-24 February 1997. This date is just a few tens of days before a visual light maximum of the AAVSO light curve, too, but three pulsation cycles before our UVES observations. With the pulsational phase at the two observations being so similar, it is hard to imagine that the photospheric temperature could be different by $200 \mathrm{~K}$, even for a Mira variable. Assuming a Li abundance constant on short time-scales, it is very unlikely that the line would go undetected in the observations of García-Hernández et al. (2007). The atmospheric structure of a Mira star certainly cannot be described perfectly with a hydrostatic model, and line strengths might vary considerably with the pulsation phase, even from cycle to cycle. It would be interesting to obtain another spectrum of RS Vir to exclude a variable Li line strength, as has been reported for a number of carbon-stars by Boffin et al. (1993).

A portion of the spectrum of RS Vir around the $670.8 \mathrm{~nm}$ Li line is displayed in Fig. 3, with the Li line clearly present. 


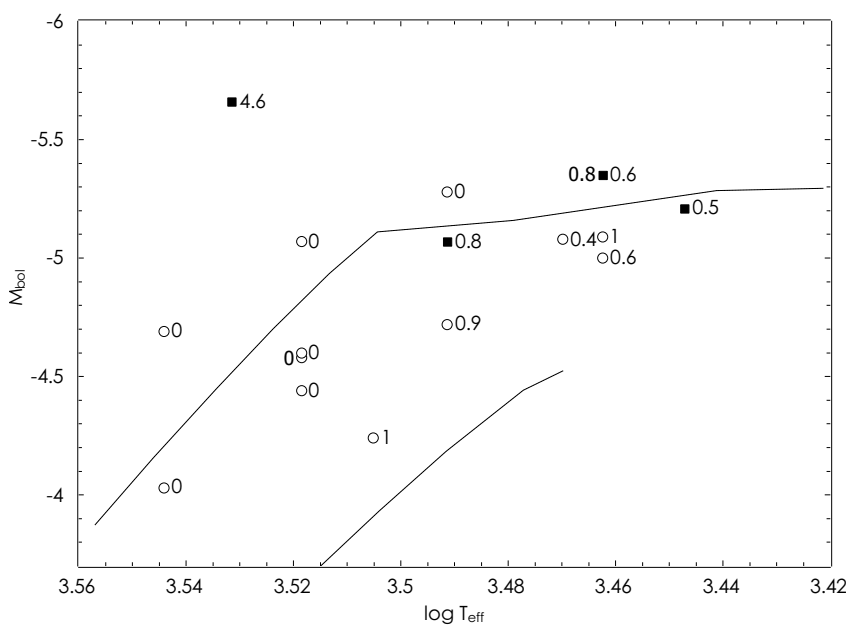

Fig. 4. $T_{\text {eff }}$ vs. $M_{\text {bol }}$ diagram for our sample stars. Filled and open symbols denote objects with and without Tc, respectively. The stars $\mathrm{S}$ Gru and R Hor overlap at $\left(M_{\mathrm{bol}}, \log T_{\text {eff }}\right)=\left(-5^{\mathrm{m}} \cdot 35,3.46\right)$. The Cstar TW Hor has been excuded from this plot. The labels give the $\mathrm{Li}$ abundance derived (see text). The solid lines are parts of isochrones of 400 Myrs age (corresponding to an AGB mass of $\sim 3 M_{\odot}$ ) and $6.3 \mathrm{Gyr}$ (AGB mass $\sim 1.05 M_{\odot}$ ), respectively, with solar metallicity and no dust formation from Marigo et al. (2008). The isochrones have been cut off once the model stars become C-rich.

\section{Discussion}

In Fig. 4 we show the location of our stars in a $T_{\text {eff }}$ vs. $M_{\text {bol }}$ diagram using the data given in Table 1, along with two isochrones from Marigo et al. (2008). Most stars nicely follow a trend of increasing luminosity with cooler temperature (higher $J-K$ ). Stars with and without Tc are marked by different symbols. In agreement with earlier studies (e.g. Lebzelter \& Hron 2003; Uttenthaler et al. 2007a), we find no Tc-rich stars below $M_{\text {bol }}=$ -4.8 . This is another confirmation of the lower luminosity limit for third dredge up predicted by various stellar evolution models. As in earlier studies, we also find here a few stars without Tc above the luminosity limit. We refer to the discussion in Lebzelter \& Hron (2003) that suggests that these stars might have too low an envelope mass for third dredge up to occur.

The data points in Fig. 4 are labelled with the derived Li-abundances from Table 2. Standard theories predict a Li abundance on the AGB below $\log \epsilon(\mathrm{Li})=1.5$. Only one star in our sample exceeds this value, namely V441 Cyg. We discuss this star in more detail in Sect. 4.1. For the other stars, there seems to be a tendency to higher Li abundance with higher luminosity or with lower temperature. This contradicts the observations of giants summarised in Michaud \& Charbonneau (1991) and Mallik (1999), as they find a decrease in the average Li abundance towards lower temperature. In Fig. 5 we show our sample, together with the results for the giants presented by Luck \& Lambert (1982) and Mallik (1999) in a $T_{\text {eff }}$ vs. $\log \epsilon(\mathrm{Li})$ plot. Our sample extends the previous dataset towards cooler temperatures. This extension reveals that the trend to decreasing $\mathrm{Li}$ abundance with lower temperature stops and may even be reversed at the cool end.

The decline in the Li abundance as a function of temperature is typically attributed to two effects (Mallik 1999): first, the surface abundance will be reduced by a deepening of the convective envelope as the stars evolve towards and up the giant branch. Second, mass loss should erode outer Li-rich layers during the advanced stages of stellar evolution.

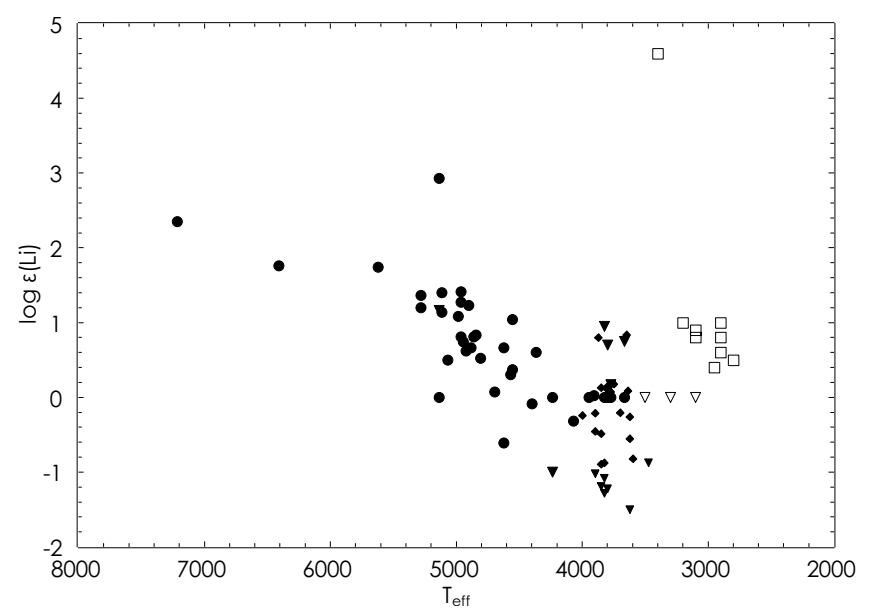

Fig. 5. Li abundance against effective temperature for the stars in our sample (open symbols) and the giants from the list of Luck \& Lambert (1982, filled diamonds) and Mallik (1999, filled circles). Large triangles represent upper limits from the sample of Mallik, small triangles those from Luck \& Lambert. The average Galactic value for the $\mathrm{Li}$ abundance is $\log \epsilon(\mathrm{Li})=3.1$ (Michaud \& Charbonneau 1991).

At the lowest luminosities we find in our sample only stars with a strong depletion of their Li abundance (see Fig. 4). These stars also have the highest temperature, thus link our sample to the one of Mallik (1999). A deep extension of the convective envelope results in the destruction of most of the Li. Lines of this element again appear in the spectrum at about $M_{\text {bol }}=-4.7$, i.e. at a slightly lower luminosity than the threshold level for Tc occurrence. This suggests that production of $\mathrm{Li}$ sets in at some point. This is supported by $\mathrm{Li}$ preferentially being detected in sample stars with long pulsation periods $(P \gtrsim 280 \mathrm{~d})$ and redder IRAS [12]-[25] colours. Based on the derived luminosities, HBB may be safely excluded as the production mechanism for all but one star.

Uttenthaler et al. (2007b) found four AGB stars in the Galactic bulge with a detectable $\mathrm{Li}$ abundance of $\log \epsilon(\mathrm{Li})$ between 0.8 and 2.0 (at an error of $0.4 \mathrm{dex}$ ) and an effective temperature around $3000 \mathrm{~K}$. These results agree nicely with our findings. Uttenthaler et al. identify extra-mixing (e.g. Boothroyd \& Sackmann 1999) as the most likely explanation for the reappearance of $\mathrm{Li}$ on the stellar surface. We speculate that the same mechanism is at work in the Li-enhanced ${ }^{3}$ stars of our sample. The lowest luminosity of a Li-enhanced star in the sample of Uttenthaler et al. (2007b) is in good agreement with the lower luminosity limit found among the targets studied here.

In this sample we cannot distinguish whether this change in $\mathrm{Li}$ abundance is coming from a difference in mass or as a result of stellar evolution or both. However, when combining our findings with the ones from Uttenthaler et al. (2007b), there seems to be a lower luminosity limit for any Li production mechanism to set in. The existence of such a limit would favour an internal mechanism over an external one (e.g. surface contamination by a companion - see Uttenthaler et al. 2007b). We note further that mass loss increases substantially during the AGB phase. As mass loss should reduce the surface $\mathrm{Li}$ abundance, the detection of an increase in Li abundance on the upper AGB strongly suggests a production of this element inside the star.

\footnotetext{
3 We use here the term "enhanced" to express a $\mathrm{Li}$ abundance above our detection threshold corresponding to $\log \epsilon(\mathrm{Li})=0$. This has to be distinguished from $\mathrm{Li}$ rich objects with an abundance exceeding the average Galactic value.
} 
Concerning a possible relation between the Li abundance and the occurrence of Tc, we find that all Tc-rich stars show also at least a mild enrichment of $\mathrm{Li}$ relative to the values found for the lowest luminosity stars of our sample. We also find Li-enhanced stars without Tc and luminous stars that show no detectable abundance of neither of the two elements. Furthermore, Uttenthaler et al. (2007b) also found Tc rich stars with no Li-enhancement. This mixture of possibilities indicates the involvement of a further parameter besides the stellar luminosity. As we have no indications of a strong scatter in metallicity in our sample, we may assume that mass is the discriminating factor. There are some indications that extra-mixing occurs more effectively among stars of lower mass, which may lead to an enhancement of surface Li in these objects (e.g. Cantiello \& Langer 2008).

If we want to compare our results with the work of Vanture et al. (2007), who find objects with all four combinations of Tc-rich, Tc-poor, Li-rich, and Li-poor, we face the problem that Vanture et al. define stars as Li-rich if $\log \epsilon(\mathrm{Li}) \geq 1.0$. All other stars are defined as Li-poor. With this approach, only two stars, RS Vir and V441 Cyg would be classified as Li rich, with the first one at the lower limit. In that sense we would find one Tc-rich/Li-rich star (see below), one Tc-poor/Li-rich star, four (five if we include TW Hor) Tc-rich/Li-poor stars and a couple of Tc-poor/Li-poor stars. This means that all four classes from Vanture et al. (2007) would be present in our sample. However, taking into account the uncertainties in the $\mathrm{Li}$ abundance and the obvious scatter of $\mathrm{Li}$ abundances between $\log \epsilon(\mathrm{Li})=0$ and 1 , we think that the Vanture et al. classes oversimplify the picture. Our understanding would certainly profit from finding an abundance limit between mildly Li-enhanced low-mass AGB stars and luminous Li-rich AGB stars producing this element by HBB in order to disentangle two different production mechanisms.

That all four combinations of Tc-rich, Tc-poor, Li-rich, and Li-poor are found can be understood at least qualitatively from the theoretical point of view. Assuming that ${ }^{3} \mathrm{He}$ is left from the RGB phase, thermohaline mixing (Cantiello \& Langer 2008) can connect the H-burning shell of a TP-AGB star with the convective envelope and thus transport ${ }^{7} \mathrm{Be}$ to cool layers where it may decay to $\mathrm{Li}$ that can be observed on the surface. This mechanism is found to be more efficient in low-mass stars $\left(\simeq 1.5 M_{\odot}\right.$, Cantiello \& Langer 2008). This scenario is not constrained to stars undergoing 3DUP events, although the faster mixing during 3DUP would ensure that even more ${ }^{7} \mathrm{Be}$ survives. In higher mass stars $\left(\gtrsim 3 M_{\odot}\right)$, thermohaline mixing cannot connect the H-burning shell with the convective envelope. Nevertheless, a ${ }^{7} \mathrm{Be}$ rich pocket can build up above the H-burning shell that can be dredged to the surface after a TP, even before formal 3DUP occurs. The ${ }^{7} \mathrm{Be}$-rich pocket would be dredged to the surface in any 3DUP event, which may account for our finding of $\mathrm{Li}$ in every Tc-rich star in the current sample. The requirement that some ${ }^{3} \mathrm{He}$ is preserved from the RGB phase also introduces a mass threshold above which extra mixing can work on the AGB, because for stars of too low an initial mass, all ${ }^{3} \mathrm{He}$ would be consumed by efficient extra-mixing already on the RGB. Thus, thermohaline mixing critically depends on the mixing history of the star.

In the magnetic buoyancy scenario (Guandalini et al. 2009), the mixing is thought to arise from magnetic bubbles with locally increased magnetic pressure, thus a decreased density. The magnetic field might be sustained by an $\alpha-\Omega$ dynamo produced by a region of rotational shear below the convective envelope. Fields of different strengths descending from different initial rotational velocities may explain the observed spread in $\mathrm{Li}$ abundances.
Independent from 3DUP, Li may be produced on the AGB by fast mixing rates (provided a ${ }^{3} \mathrm{He}$ reservoir is still present from the RGB), or destroyed by slow mixing rates.

Quantitative predictions of models of extra mixing are still rather uncertain and require parameter fine-tuning by observations. Depending on the adopted efficiency parameter of thermohaline mixing, Cantiello \& Langer (2008) predict a surface $\mathrm{Li}$ enrichment of $\sim 0.05$ dex during one inter-pulse period of a $3 M_{\odot}$ TP-AGB model star. After a number of 3DUP events, the surface $\mathrm{Li}$ abundance may thus increase above the detection threshold in a cool atmosphere. Thermohaline mixing is expected to be more efficient in lower mass stars, so the surface $\mathrm{Li}$ abundance could be increased by larger amounts than for the described case of a $3 M_{\odot}$ star. It should be noted that the interaction of thermohaline mixing with magneto-rotational instabilities is important, but only full magneto-hydrodynamical simulations can lead to a better understanding of these interactions.

The magnetic buoyancy picture, on the other hand, predicts an upper limit on Li production at the level of $\log \epsilon(\mathrm{Li})=2-2.5$. This upper limit is set by assuming a short phase of very fast mixing (a few $\mathrm{km} \mathrm{s}^{-1}$ ) of magnetised bubbles, in which no burning of the material occurs, and the Li enrichment of the envelope is set by the amount of "normal" ${ }^{7} \mathrm{Be}$ that is produced near the H-burning shell. The Li abundances below this upper limit can be easily reproduced by assuming some Li destruction by a slower transport of the material, such that some nuclear burning occurs during the transfer to the envelope. If magnetic fields are the origin of the extra mixing, both fast and slow regimes of circulation can explain very different outcomes with only a single physical mechanism.

To conclude, super-Li rich stars such as V441 Cyg cannot be easily explained by the magnetic buoyancy scenario, but the rest of our sample would be described well. To decide whether thermohaline mixing could be the dominant mechanism, more information on the masses and mixing histories of the stars would be required.

We end this discussion with a closer look onto two of our sample stars. As far as we know, the reclassification of W Eri as Tc-poor has no great consequences on understanding the nature of this star. However, the re-classification of V441 Cyg and R Hya as Tc-rich and Tc-poor, respectively, has important consequences, and we thus devote the following two sections to these two objects.

\subsection{V441 Cygni}

Even though the SNR of the spectrum of V441 Cyg is low, it is clear from Fig. 2 that this star indeed has Tc in its atmosphere. It is thus an intrinsic S-star. As further evidence, its spectrum around the three strongest Tc lines is shown in Fig. 6. Also Wang \& Chen (2002) list V441 Cyg as "not a good candidate Tc-deficient S star" based on its near- and mid-IR photometry.

Vanture et al. (2007) investigate a sample of 59 Galactic $\mathrm{S}$-stars for the correlation of $\mathrm{Tc}$ and $\mathrm{Li}$ and find all four possible combinations of Tc-rich and -poor, and Li-rich and -poor in their sample. However, the group of Tc-poor, Li-rich S-stars is formed by only one object, namely V441 Cyg. Vanture et al. (2007) thus call it a "unique" object. There was good reason for doubt because V441 Cyg actually has never been checked for its Tc content in the literature, hence our FOCES observations. As shown above, there is no Li-rich (not Li-enhanced!) and Tc-poor S-type star known at the moment.

We thus show that V441 Cyg is Tc- and Li-rich and that it needs to be moved to "group 2" as defined by 

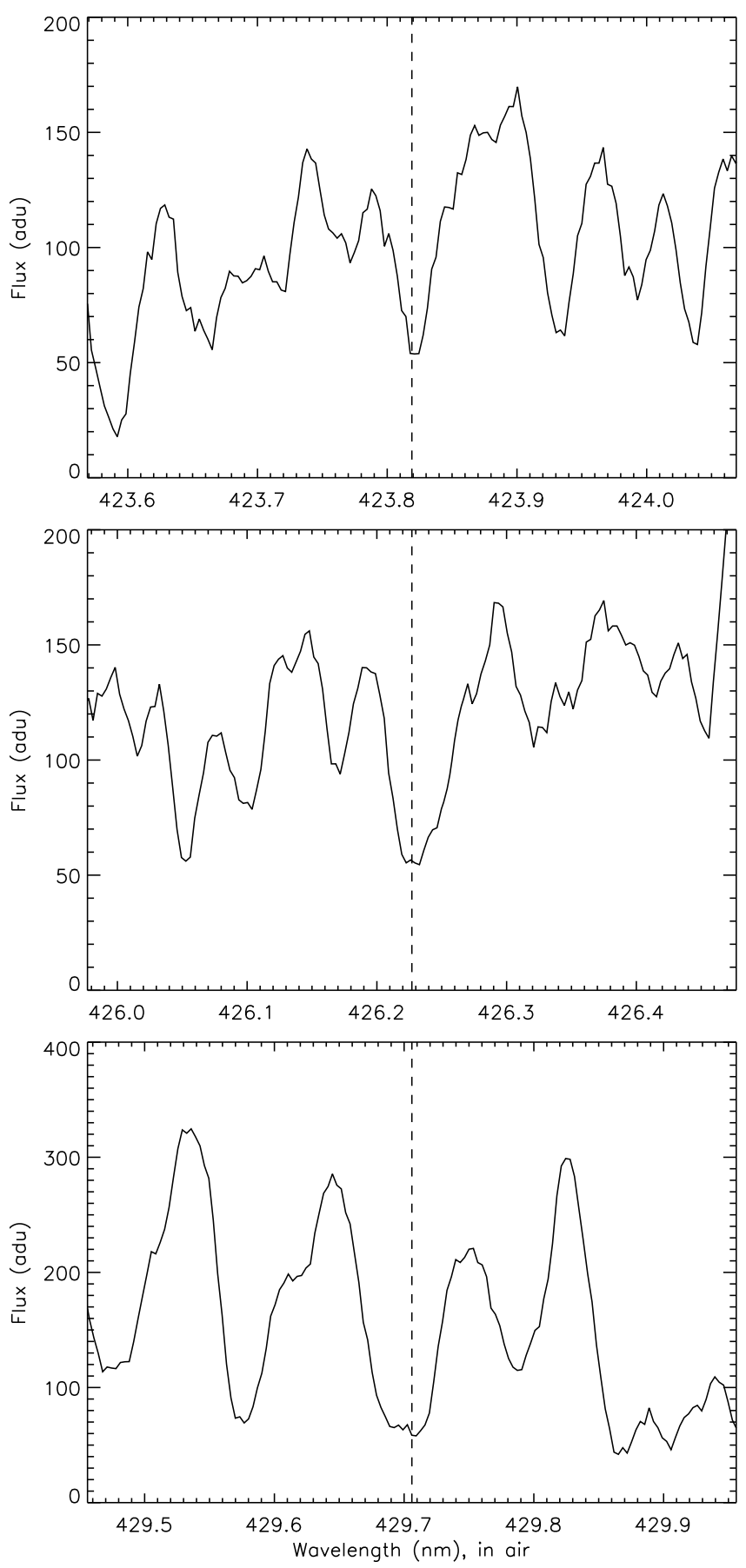

Fig. 6. FOCES spectrum of V441 Cyg around the three strongest TcI lines. The spectrum has been smoothed with a 5 pixel boxcar. The dashed line marks the laboratory wavelength of the respective line. Clearly, all three TcI lines are present.

Vanture et al. (2007). They offer two possibilities for interpreting this group of stars. The first interpretation is that intrinsic (Tc-rich) S-stars with high Li-abundance are intermediate-mass $\left(M \gtrsim 4 M_{\odot}\right)$ TP-AGB stars experiencing $\mathrm{HBB}$, thus producing $\mathrm{Li}$ in their atmospheres and destroying $\mathrm{C}$ via $\mathrm{CN}$ cycling. This picture is supported by the low average Galactic latitude of the stars in group $2\left(14^{\circ}\right.$, excluding two relatively nearby stars). However, the bolometric magnitude, which is available for only four stars of the Vanture et al. (2007) sample, instead argues against this interpretation, since none of them has $M_{\mathrm{bol}} \leq-6^{\mathrm{m}} 0$. Vanture et al. (2007) thus suggest that their sample consists of a mix of low- and intermediate-mass stars. The second interpretation thus concerns low-mass S-stars, which might be the result of extra-mixing processes on the TP-AGB (see discussion above). The existence of Li-rich C-stars (Boffin et al. 1993) suggests that low-mass Li-rich S-stars could also exist, and have not yet dredged-up enough carbon to reach $\mathrm{C} / \mathrm{O}>1$.

Which of the two offered interpretations holds for V441 Cyg? It is located at a very low Galactic latitude of $b \sim-1^{\circ}$, and our estimate of its bolometric magnitude $\left(M_{\text {bol }}=-5^{m} \cdot 66\right)$ is not far below the approximate limit for HBB. Concerning the uncertainties in determining the luminosity we note that only if the star were be a fundamental mode pulsator, we would expect a lower luminosity. This definitely argues in favour of the intermediate-mass HBB interpretation. However, García-Hernández et al. (2007) find that Li-rich Galactic OH/IR stars, thought to be intermediate-mass AGB stars with a very high mass-loss rate, do not exhibit significant $\mathrm{Zr}$ enhancements. On the other hand, the s-element $\mathrm{Rb}$ is observed to be enriched in these stars (García-Hernández et al. 2006). This might stem from the activation of the ${ }^{22} \mathrm{Ne}(\alpha, \mathrm{n})^{25} \mathrm{Mg}$ neutron source rather than the ${ }^{13} \mathrm{C}(\alpha, \mathrm{n}){ }^{16} \mathrm{O}$ neutron source in these more massive stars. Thus, enrichment in $\mathrm{Rb}$ is another indicator of high mass. Unfortunately, we did not succeed to derive a reliable $\mathrm{Rb}$ abundance from our spectrum of V441 Cyg, because the Rb resonance line at $780.0 \mathrm{~nm}$ is completely dominated by a blue-shifted circumstellar component that makes it impossible to determine the photospheric Rb abundance. Nevertheless, we can interpret this as no enhancement of Rb in the photosphere of V441 Cyg, suggesting that it is too low in mass to activate the ${ }^{22} \mathrm{Ne}$ neutron source. The light curve of V441 Cyg changes with a period of $288 \mathrm{~d}$ in a semi-regular way, and the IRAS [12]-[25] colour, defined as $-2.5 \log \left(F_{12} / F_{25}\right)$, has a value of -1.28 . Only three of our sample stars are bluer than that, which suggests only low obscuration by the circumstellar envelope. The variability characteristics, in particular the low amplitude of pulsation at this high luminosity, could be interpreted as a consequence of a high stellar mass, but is difficult to quantify.

To summarise, the evidence on the mass of V441 Cyg is not entirely conclusive, and we cannot clearly decide on which of the interpretations offered by Vanture et al. (2007) holds for this star. There are a number of other peculiar intrinsic S-stars with high Li abundance, e.g. R And and T Sgr. The Rb abundance has been measured in the former by Wallerstein (1992), and a neutron density at the s-process site of $10^{8} \mathrm{~cm}^{-1}$ was derived, indicative for the ${ }^{13} \mathrm{C}$ neutron source operating in low-mass stars. An analysis and comparison of these stars would be be very rewarding, which we plan to present in a forthcoming paper.

Finally, Fig. 7 reproduces a small piece of spectrum of V441 Cyg around the $670.8 \mathrm{~nm}$ Li resonance line, along with the best-fitting synthetic spectrum based on a MARCS model atmosphere with $T_{\text {eff }}=3400 \mathrm{~K}$ and solar metallicity, assuming $\log \epsilon(\mathrm{Li}) \simeq+4.6$. There is a prominent blue wing in the $\mathrm{Li}$ line, which we did not try to fit. This feature may be interpreted as indication of a Li-rich stellar wind that cannot be reproduced with our hydrostatic models. Such a wind feature is somewhat surprising, given the rather blue IRAS colours of this star. Because the temperature is probably lower in the wind than in the photosphere, more Li atoms are present in the ground state, and the wing is deeper than the photospheric line component. Thus, we possibly see V441 Cyg in a stage where it enriches the ISM with freshly produced Li. 


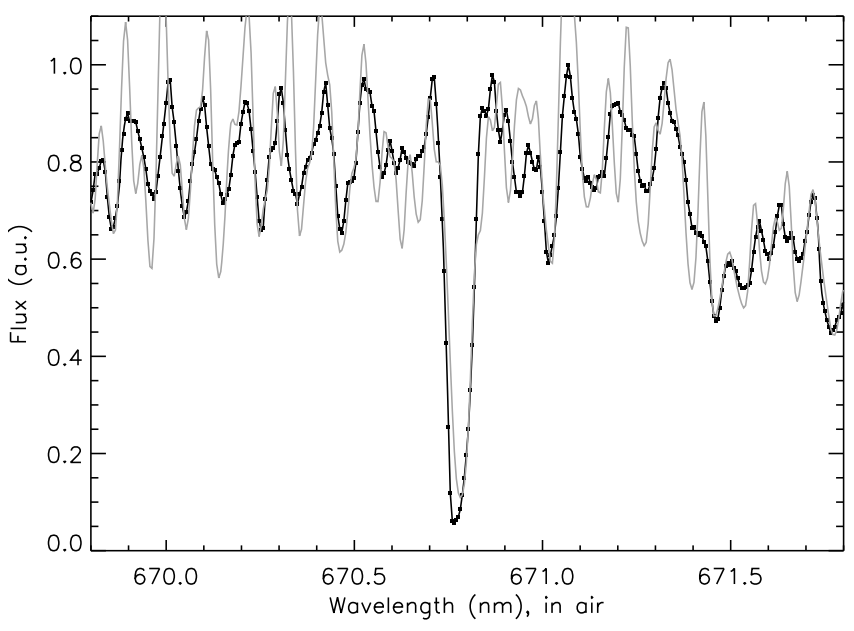

Fig. 7. FOCES spectrum of V441 Cyg around the $670.8 \mathrm{~nm} \mathrm{Li} \mathrm{res-}$ onance line (black graph with squares), and a best fitting synthetic spectrum (grey graph) based on a MARCS model atmosphere with $T_{\text {eff }}=3400 \mathrm{~K}$ and solar metallicity. A Li abundance of $\log \epsilon(\mathrm{Li}) \simeq+4.6$ has been assumed in the spectral synthesis. We did not try to fit the blue wing of the Li line, because we interpret it to be caused by a Li-rich stellar wind from V441 Cyg.

\subsection{R Hya}

$\mathrm{R}$ Hya has already been classified as Tc-rich a long time ago, albeit with only a low Tc line intensity (Merrill 1952b). Spectrogram tracings of R Hya taken at different phases of its pulsation cycle can be found in Merrill (1952a). These spectrograms cover the Tc I lines at 398.497 and $404.911 \mathrm{~nm}$, but both are absent at all observed pulsational phases. Also in our highquality, high-resolution UVES spectra of R Hya, we are unable to detect any of the Tc lines. We find no increase in $\mathrm{ZrO}$ band strength, wether in R Hya or in any other Tc-poor star in our sample. We thus conclude that, with the spectrograms available at the times of Merrill, a precise decision about the presence of weak absorption lines was not possible, and a misidentification occurred in this particular case that has continued in the literature ever since. We find R Hya to be the most luminous Tc-poor star in our sample, with approximately 10000 solar luminosities. Because of its Mira type variability with a rather long pulsation period, as well as its slightly redder IRAS colours, it is probably in a more advanced AGB stage than other suspected truly low-mass AGB stars, such as ER Vir and EV Vir.

$\mathrm{R}$ Hya is of particular interest because its pulsation period has strongly decreased in time (Templeton et al. 2005). It is thus speculated that it has recently undergone a TP (Wood \& Zarro 1981). If this interpretation of the period change is correct, then either the most recent thermal pulse was not followed by a third dredge-up event, or the dredged-up inter-shell material has not yet reached the surface of the star. The absence of Tc in the atmosphere of R Hya, however, does not exclude the possibility that a recent TP is indeed the cause of the observed period decrease.

From detailed AGB evolution models (Cristallo et al. 2009), it is known that a 3DUP episode, if it takes place, follows a TP with some time delay. For instance, for a $2 M_{\odot}$ model of solar metallicity, the first 3DUP episode takes place $\sim 670$ years after the preceeding TP (Cristallo, private communication). Thus, abundance changes of s-process elements and ${ }^{12} \mathrm{C}$ on the stellar surface also become visible only with a time delay. Wood \& Zarro (1981) suggest that a TP could have occurred in R Hya about 550 years ago. If the decrease in pulsation period observed in R Hya was indeed caused by a recent TP, we may be able to detect surface abundance changes (e.g. Tc) in the future, say in a few 100 years, if this past TP is followed by a 3DUP event ${ }^{4}$.

Alternatively, Zijlstra et al. (2002) suggest that the period decrease observed in R Hya could just as well be caused by a nonlinear instability leading to an internal relaxation of the stellar structure. While not detecting Tc does not strongly favour one or the other scenario, it indicates that possible TPs are not strong enough to drive 3DUP in R Hya.

\section{Summary and conclusions}

We analysed high-resolution optical spectra of a sample of 17 M-type (oxygen-rich) and one C-type (carbon-rich) AGB variables for the presence of Tc and the abundance of $\mathrm{Li}$. We employed a flux-ratio method to decide on the presence of the 3rd dredge-up indicator Tc in the stellar atmosphere, and measured the abundance of the temperature-sensitive element Li with spectral synthesis techniques of the $670.8 \mathrm{~nm} \mathrm{Li} \mathrm{reso-}$ nance line using MARCS model atmospheres. Bolometric magnitudes were established from near-IR photometry and pulsation periods. The lower luminosity limit for 3DUP to occur, established in previous works, has been confirmed. We re-classified three sample stars with respect to their Tc content, namely that R Hya and W Eri are Tc-poor, and V441 Cyg is Tc-rich. We thus suggest that R Hya has not yet experienced a dredge-up of freshly produced s-elements, but, regarding the possibility that it recently underwent a TP, might have a 3DUP episode in the future. V441 Cyg, on the other hand, is an intrinsic S-star.

Lithium is detected in ten sample stars (with a $\log \epsilon(\mathrm{Li})>0$ ). After combining these abundances with bolometric magnitude estimates, we find that $\mathrm{Li}$ is more abundant in more luminous and cool stars. All Tc-rich sample stars are found to contain also some $\mathrm{Li}$ in their atmosphere. We thus suggest that this fragile element could be replenished in the stellar atmosphere by some (extra-mixing) process that is only active in the more luminous objects. This could also be connected to a mass effect within the sample. The S-star V441 Cyg is found to be super-Li rich, with a higher Li abundance than the solar photospheric value probably by a factor 1000 . The gathered evidence does not allow us to clearly conclude whether this object is an intermediate-mass AGB star $\left(M \gtrsim 4 M_{\odot}\right)$ producing Li by the HBB mechanism, or a low-mass star with extremely efficient extra-mixing processes. The data obtained in this study may be useful for constraining theoretical models of such extra-mixing processes.

Acknowledgements. We thank M. Busso and M. Cantiello for their fruitful comments, M. T. Lederer for constructing the model atmospheres used in this study, J. Hron for carrying out the visitor-mode observations with UVES/VLT, the Calar Alto Observatory staff for carrying out the service mode observations of V441 Cyg, and the anonymous referee for useful comments. SU acknowledges support from the Fund for Scientific Research of Flanders (FWO) under grant number G.0470.07. TL acknowledges support by the Austrian Science Fund FWF under project number P20046-N16. This publication makes use of data products from the Two Micron All Sky Survey, which is a joint project of the University of Massachusetts and the Infrared Processing and Analysis Center/California Institute of Technology, funded by the National Aeronautics and Space Administration and the National Science Foundation. We acknowledge with thanks the variable star observations from the AAVSO International Database contributed by observers worldwide and used in this research.

\footnotetext{
4 For the s-process elements to be enhanced, it takes at least two 3DUP episodes, one to build up a ${ }^{13} \mathrm{C}$ pocket in the inter-shell region as neutron source, and another one to actually mix the s-process products to the surface. C enhancement should be present after only one 3DUP episode.
} 


\section{References}

Aringer, B. 2000, The SiO Molecule in the Atmospheres and Circumstellar Shells of AGB Stars, Ph.D. Thesis, Univ. of Vienna, Austria

Boffin, H. M. J., Abia, C., Isern, J., \& Rebolo, R. 1993, A\&AS, 102, 361

Boothroyd, A. I., \& Sackmann, I.-J. 1999, ApJ, 510, 232

Busso, M., Gallino, R., \& Wasserburg, G. J. 1999, ARA\&A, 37, 239

Busso, M., Wasserburg, G. J., Nollett, K. M., \& Calandra, A. 2007, ApJ, 671, 802

Carpenter, J. M. 2001, AJ, 121, 2851

Cameron, A. G. W., \& Fowler, W. A. 1971, ApJ, 164, 111

Cantiello, M., \& Langer, N. 2008, IAUS, 252, 103 (The Art of Modeling Stars in the 21st Century)

Catchpole, R. M., Robertson, B. S. C., Lloyd Evans, T. H. H., et al. 1979, SAAOC, 1,61

Charbonnel, C., \& Balachandran, S. C. 2000, A\&A, 359, 563

Charbonnel, C., \& Zahn, J.-P. 2007, A\&A, 467, 15

Cristallo, S., Straniero, O., Lederer, M. T., \& Aringer, B. 2007, ApJ, 667, 489

Cristallo, S., Straniero, O., Gallino, R., et al. 2009, ApJ, 696, 797

de la Reza, R., Drake, N. A., \& da Silva, L. 1997, ApJ, 482, L77

Denn, G. R., Luck, R. E., \& Lambert, D. L. 1991, ApJ, 377, 657

Dominy, J. F., \& Wallerstein, G. 1986, ApJ, 310, 371

Eggleton, P. P., Dearborn, D. S. P., \& Lattanzio, J. C. 2006, Science, 314, 1580

Fouquet, P., Le Bertre, T., Epchtein, N., Gugliemo, F., \& Kerschbaum, F. 1992, A\&AS, 93, 151

García-Hernández, D. A., García-Lario, P., Plez, B., et al. 2006, Science, 314, 1751

García-Hernández, D. A., García-Lario, P., Plez, B., et al. 2007, A\&A, 462, 711

Gilroy, K. K., \& Brown, J. A. 1991, ApJ, 371, 578

Guandalini, R., \& Busso, M. 2008, A\&A, 488, 675

Guandalini, R., Busso, M., Palmerini, S., \& Uttenthaler, S. 2009, PASA, in press

Henden, A. A., 2009, Observations from the AAVSO International Database, priv. comm.

Herwig, F. 2005, ARA\&A, 43, 435

Iben, I. 1973, ApJ 185, 209

Ita, Y., Tanabé, T., Matsunaga, N., et al. 2004, MNRAS, 347, 720

Jørgensen, U. G., Johnson, H. R., \& Nordlund, A. 1992, ASPC, 26, 540

Kerschbaum, F. 1995, A\&AS, 113, 441

Kerschbaum, F., \& Hron, J. 1994, A\&AS, 106, 397

Kholopov, P. N., Samus, N. N., Frolov, M. S., et al. 1985-1988, General Catalogue of Variable Stars, 4th edn. (Moscow: Nauka Publishing House)

Kiss, L. L., \& Bedding, T. R. 2003, MNRAS, 343, L79

Kipper, T. 2004, Baltic Astron., 13, 573

Korn, A., Grundahl, F., Richard, O., et al. 2006, Nature, 442, 657

Kupka, F., Piskunov, N., Ryabchikova, T. A., Stempels, H. C., \& Weiss, W. W. 1999, A\&AS, 138, 119

Lambert, D. L., Smith, V. V., \& Heath, J. 1993, PASP, 105, 568
Lebzelter, T., \& Hron, J. 1999, A\&A, 351, 533

Lebzelter, T., \& Hron, J. 2003, A\&A, 411, 533

Lebzelter, T., Hinkle, K. H., Wood, P. R., Joyce, R. R., \& Fekel, F. C. 2005, A\&A, 431, 623

Lebzelter, T., Lederer, M. T., Cristallo, S., et al. 2008, A\&A, 486, 511

Lenz, P., \& Breger, M. 2005, Comm. Asteroseism., 146, 53

Little, S. J., Little-Marenin, I. R., \& Hagen-Bauer, W. 1987, AJ, 94, 981

Luck, R. E., \& Lambert, D. L. 1982, ApJ, 256, 189

McKellar, A. 1940, PASP, 52, 407

Mallik, S. V. 1999, A\&A, 352, 495

Marigo, P., Girardi, L., Bressan, A., et al. 2008, A\&A, 482, 883

Mekul, L. 2005, diploma Thesis, University of Vienna

Merrill, P. W. 1952a, ApJ, 116, 18

Merrill, P. W. 1952b, ApJ, 116, 21

Michaud, G., \& Charbonneau, P. 1991, Space Sci. Rev., 57, 1

Nollett, M. N., Busso, M., \& Wasserburg, G. J. 2003, ApJ, 582, 1036

Nordlund, A. 1984, in Methods in Radiative Transfer, ed. W. Kalkofen (Cambridge: Cambridge University Press), 211

Ochsenbein, F., Bauer, P., \& Marcout, J. 2000, A\&AS, 143, 221

Pfeiffer, M. J., Frank, C., \& Baumüller, D., et al. 1998, A\&AS, 130, 381

Plez, B. 1998, A\&A, 337, 495

Plez, B., van Eck, S., Jorissen, A., et al. 2003, IAUS, 210, A2

Pojmański, G., Pilecki, B., \& Szczygiel, D. 2005, Acta Astron., 55, 275

Rebolo, R. 1991, IAUS, 145, 85 (Evolution of Stars: The Photospheric

Abundance Connection)

Reyniers, M., Van Winckel, H., Biémont, E., \& Quinet, P. 2002, A\&A, 395, L35

Samus, N. N., Durlevich, O. V., et al. 2004, Combined General Catalogue of

Variable Stars, VizieR On-line Data Catalog: II/250

Schwenke, D. 1998, Faraday Discuss., 109, 321

Skrutskie, M. F., Cutri, R. M., Stiening, R., et al. 2006, AJ, 131, 1163

Smith, V. V., Plez, B., Lambert, D. L., \& Lubowich, D. A. 1995, ApJ, 441, 735

Spite, M., \& Spite, F. 1982, Nature, 297, 483

Templeton, M. R., Mattei, J. A., \& Willson, L. A. 2005, AJ, 130, 776

Torres-Peimbert, S., Wallerstein, G., \& Phillips, J. G. 1964, ApJ, 140, 1313

Uttenthaler, S., Hron, J., Lebzelter, T., et al. 2007a, A\&A, 463, 251

Uttenthaler, S., Lebzelter, T., Palmerini, S., et al. 2007b, A\&A, 471, L41

Van Eck, S., \& Jorissen, A. 1999, A\&A, 345, 127

Vanture, A. D., \& Wallerstein, G. 2002, ApJ, 564, 395

Vanture, A. D., Smith, V. V., Lutz, J., et al. 2007, PASP, 119, 147

Wang, X.-H., \& Chen, P.-S. 2002, A\&A, 387, 129

Wallerstein, G. 1992, PASP, 104, 511

Wallerstein, G., Iben, I., Parker, P., et al. 1997, Rev. Mod. Phys., 69, 995

Wasserburg, G. J., Boothroyd, A. I., \& Sackmann, I.-J. 1995, ApJ, 447, L37

Wood, P. R., \& Zarro, D. M. 1981, ApJ, 247, 247

Wood, P. R., Alcock, C., Allsman, R. A., et al. 1999, IAUS, 191, 151

Zijlstra, A. A., Bedding, T. R., \& Mattei, J. A. 2002, MNRAS 334, 498 